\title{
Screening of FOXD3 targets in lung cancer via bioinformatics analysis
}

\author{
WENHUA JIANG ${ }^{1}$, PENGFEI LIU ${ }^{2-6}$ and XIAODONG LI ${ }^{1}$ \\ ${ }^{1}$ Department of Radiotherapy, The Second Hospital of Tianjin Medical University, Tianjin 300211; ${ }^{2}$ Department of \\ Lymphoma, ${ }^{3}$ National Clinical Research Center for Cancer, ${ }^{4}$ Key Laboratory of Cancer Prevention and Therapy, \\ ${ }^{5}$ Tianjin's Clinical Research Center for Cancer, ${ }^{6}$ Sino-US Center of Lymphoma and Leukemia, \\ Tianjin Medical University Cancer Institute and Hospital, Tianjin 300060, P.R. China
}

Received March 23, 2017; Accepted November 2, 2017

DOI: $10.3892 / \mathrm{ol} .2017 .7685$

\begin{abstract}
The purpose of the present study was to explore the targets of forkhead box D3 (FOXD3) in lung cancer, and thus contribute to the diagnosis and therapy of the disease. The gene expression profile of GSE64513 was downloaded from the Gene Expression Omnibus database. The dataset contained 3 FOXD3 knockout A549 lung cancer cell samples and 3 normal A549 cell samples. The differentially expressed genes (DEGs) between the FOXD3-knockout and normal A549 cells were identified using the limma package in $\mathrm{R}$. The alternative splicing genes (ASGs) in FOXD3-knockout samples were identified by Replicate Multivariate Analysis of Transcript Splicing software. The Database for Annotation, Visualization and Integrated Discovery was used to identify the enriched functions and pathways of DEGs and ASGs. A protein-protein interaction (PPI) network was constructed based on results from the Search Tool for the Retrieval of Interacting Genes database and visualized using Cytoscape software. A total of 1,853 DEGs and 2,249 ASGs were identified in FOXD3-knockout A549 cells compared with normal A549 cells. The DEGs were enriched in 338 Gene Ontology (GO) terms and 21 Kyoto Encyclopedia of Genes and Genomes (KEGG) pathways, and the ASGs were enriched in 470 GO terms and 22 KEGG pathways. A total of 199 overlaps between the DEGs and the ASGs were identified; a PPI network constructed based on the overlapping genes contained 97 nodes and 115 pairs. FOXD3 may serve an important role in regulating the growth, migration and proliferation of tumor cells in lung cancer. The present study indicates that a number of genes, including AURKA and NOS3, may be targets of $F O X D 3$, mediating its effect in lung cancer.
\end{abstract}

Correspondence to: Dr Xiaodong Li, Department of Radiotherapy, The Second Hospital of Tianjin Medical University, 23 Pingjiang Road, Tianjin 300211, P.R. China

E-mail: xiaodonglitj@163.com

Key words: lung cancer, forkhead box D3, Gene Expression Omnibus database, bioinformatics

\section{Introduction}

Lung cancer is among the most common types of cancer, accounting for $\sim 13 \%$ of all cancer cases (1). The generally poor prognosis of lung cancer renders it a leading cause of cancer-associated mortality worldwide (2). In 2010, 1.5 million mortalities due to lung cancer were reported, representing $19 \%$ of all cancer-associated mortality (3). The incidence of lung cancer has doubled in China over the past decade due to issues including the aging population, smoking and the reduced air quality (4). Lung cancer is initiated by the activation of oncogenes or the inactivation of tumor suppressor genes (5). Despite advances in diagnosis and treatment, the prognosis of lung cancer remains relatively poor. The identification of reliable biomarkers and novel genes involved in lung cancer carcinogenesis is important for improving the ability to predict the prognosis and to guide the therapy of lung cancer.

Forkhead box D3 (FOXD3) is a member of the FOX transcription factor family, which is characterized by a distinct forkhead domain (6). FOXD3 acts as a transcriptional repressor or activator (7). The abnormal expression of FOXD3 has been reported to participate in tumor onset and progression in non-small cell lung cancer tumor cells (8). Other studies have indicated tumor suppressive activities for FOXD3, including the inhibition of cell growth and invasion in various types of cancer, including gastric cancer and melanoma $(9,10)$. A number of genes associated with tumorigenesis have been reported to be targets of FOXD3. One study demonstrated that $F O X D 3$ regulated $R N D 3$ expression and migration properties in melanoma cells (11). Another reported that FOXD3 exhibited tumor suppressive activity that affected the growth, aggressiveness and angiogenesis of neuroblastoma through the transcriptional regulation of NDRG1 (12). However, the role of FOXD3 in lung cancer remains uncharacterized.

In this study, differentially expressed genes (DEGs) and alternative splicing genes (ASGs) were identified in FOXD3-knockout samples compared with normal samples. Functional and pathway enrichment analyses of the DEGs and ASGs were performed. A protein-protein interaction (PPI) network was constructed based on the overlaps between the 
DEGs and ASGs. An improved understanding of FOXD3 in regulating the process of lung cancer was obtained, which may allow the development of novel strategies for the diagnosis and therapy of lung cancer.

\section{Materials and methods}

Datasets. The gene expression profile GSE64513 was downloaded from the Gene Expression Omnibus (GEO; http://www. ncbi.nlm.nih.gov/geo/) database. The data set contained the RNAseq data from 6 samples, including 3 FOXD3-knockout A549 lung cancer cell samples and 3 normal A549 cell samples.

Screening of DEGs and ASGs. The data was first analyzed using FastQC (http://www.bioinformatics.babraham.ac.uk/projects/fastqc), a java-based high-throughput data quality control software. Reads with base quality scores $<20$ were discarded, and reads longer than $30 \mathrm{bp}$ were selected for further investigation. The remaining reads were mapped to the GRCh37/hg19 genome based on the Tophat 2 program (13). The number of reads mapped to the exons of each gene was counted with the HTSeq-Count tool (14) and regarded as the expression profile of each gene. Differently expressed genes (DEGs) in FOXD3 knockout lung cancer samples compared with normal samples were identified using the edge R package (15) with the following thresholds: False discovery rate $<0.01$ and $\log$ (fold change) $\mid>1$. The hierarchical clustering of DEGs was performed using the heatmap. 2 function of the gplots package in Various R Programming tool (version 2.12) (16). The alternative splicing genes (ASGs) in the FOXD3 knockout samples were identified using the replicate multivariate analysis of transcript splicing (rMATS) program, a computer program designed to detect differential alternative splicing from replicate RNA-Seq data (17).

Functional and pathway enrichment analysis. The Database for Annotation, Visualization and Integrated Discovery (DAVID; https://david.ncifcrf.gov/) is a web-based tool for genomic functional annotations (18). To further explore the biological functions of the DEGs and ASGs, Gene Ontology (GO) and Kyoto Encyclopedia of Genes and Genomes (KEGG) enrichment analyses were performed using DAVID, with the threshold of $\mathrm{P}<0.05$.

Construction of a PPI network. The overlapping DEGs and ASGs were analyzed using the Search Tool for the Retrieval of Interacting Genes (STRING; http://string-db.org/) $(19,20)$. A PPI network to illustrate the identified interactions was constructed and visualized using Cytoscape 3.4 (21).

\section{Results}

Identification of DEGs and ASGs. The total number of reads, the number of mapped reads and the mapping rate of each sample is provided in Table I. A total of 1,853 DEGs were identified, of which 382 were upregulated and 1,471 were downregulated. The top 20 DEGs are listed in Table II. Fig. 1 demonstrates the hierarchical clustering results for each sample graphically (Fig. 1A), the fold-change trend of the expression
Table I. Total reads, the number of mapped reads, and the mapping rates of each sample.

\begin{tabular}{lccc}
\hline Sample & Total reads & Mapped reads & Mapping rate, \% \\
\hline SRR1734826 & $10,339,232$ & $9,252,784$ & 89.5 \\
SRR1734827 & $10,472,212$ & $9,298,751$ & 88.8 \\
SRR1734828 & $10,868,010$ & $9,651,798$ & 88.8 \\
SRR1734829 & $11,224,483$ & $9,666,662$ & 86.1 \\
SRR1734830 & $10,548,877$ & $9,241,415$ & 87.6 \\
SRR1734831 & $11,578,464$ & $10,104,885$ & 87.3 \\
\hline
\end{tabular}

Table II. Top 20 differentially expressed genes of the forkhead Box D3-knockout lung cancer A549 cell samples compared with normal A549 cells.

\begin{tabular}{lccc}
\hline $\begin{array}{l}\text { Gene } \\
\text { symbol }\end{array}$ & $\begin{array}{c}\text { False discovery } \\
\text { rate }\end{array}$ & P-value & $\begin{array}{c}\text { Log fold } \\
\text { change }\end{array}$ \\
\hline SAA1 & $1.02 \times 10^{-426}$ & $8.46 \times 10^{-313}$ & -7.70213 \\
C3 & $3.82 \times 10^{-478}$ & $8.15 \times 10^{-562}$ & -2.97374 \\
GAS6 & $9.26 \times 10^{-283}$ & $2.01 \times 10^{-286}$ & -2.52365 \\
CFB & $4.97 \times 10^{-271}$ & $1.44 \times 10^{-274}$ & -3.25002 \\
LCN2 & $7.38 \times 10^{-254}$ & $2.67 \times 10^{-257}$ & -3.10027 \\
TGM2 & $8.36 \times 10^{-244}$ & $3.63 \times 10^{-247}$ & -2.45302 \\
SAT1 & $3.53 \times 10^{-238}$ & $1.79 \times 10^{-241}$ & -2.46616 \\
PDZK1IP1 & $3.63 \times 10^{-230}$ & $2.11 \times 10^{-233}$ & -4.94302 \\
PLAU & $7.60 \times 10^{-226}$ & $4.96 \times 10^{-229}$ & -2.07647 \\
SAA2 & $6.42 \times 10^{-194}$ & $4.65 \times 10^{-197}$ & -7.47593 \\
TNIP1 & $2.23 \times 10^{-189}$ & $1.78 \times 10^{-192}$ & -2.05325 \\
SPP1 & $2.23 \times 10^{-189}$ & $1.94 \times 10^{-192}$ & 2.29076 \\
S100A8 & $1.27 \times 10^{-168}$ & $1.20 \times 10^{-171}$ & -3.79768 \\
TMEM132A & $1.42 \times 10^{-166}$ & $1.44 \times 10^{-169}$ & -1.93297 \\
ASNS & $6.48 \times 10^{-156}$ & $7.04 \times 10^{-159}$ & -2.09409 \\
SERPINE1 & $2.92 \times 10^{-149}$ & $3.38 \times 10^{-152}$ & -2.55607 \\
PHLDB2 & $4.19 \times 10^{-147}$ & $5.17 \times 10^{-150}$ & -1.54635 \\
ICAM1 & $7.56 \times 10^{-144}$ & $9.87 \times 10^{-147}$ & -2.03788 \\
CXCL8 & $1.08 \times 10^{-138}$ & $1.48 \times 10^{-141}$ & -5.25318 \\
\hline & & & \\
\hline
\end{tabular}

of the identified DEGs (Fig. 1B) and the hierarchical cluster analysis of the samples based on the DEGs (Fig. 1C). A total of 2,249 genes with alternative splicing were identified in FOXD3-knockout lung cancer samples compared with normal A549 cell samples, including 545 with an alternative 3 ' splice site, 412 with an alternative 5' splice site, 1,629 with mutually exclusive exons and 67 with retained introns.

Enriched GO terms and KEGG pathways of DEGs and ASGs. The DEGs were enriched in $338 \mathrm{GO}$ terms and $21 \mathrm{KEGG}$ pathways. The ASGs were enriched in $470 \mathrm{GO}$ terms and 22 KEGG pathways. The top $10 \mathrm{GO}$ terms for the ASGs and DEGs are listed in Fig. 2A and B, respectively. Table III lists the enriched KEGG pathways for the ASGs and DEGs. The DEGs were predominately enriched in 'graft-vs.-host disease', 'hematopoietic cell lineage', 'ECM-receptor interaction' and 

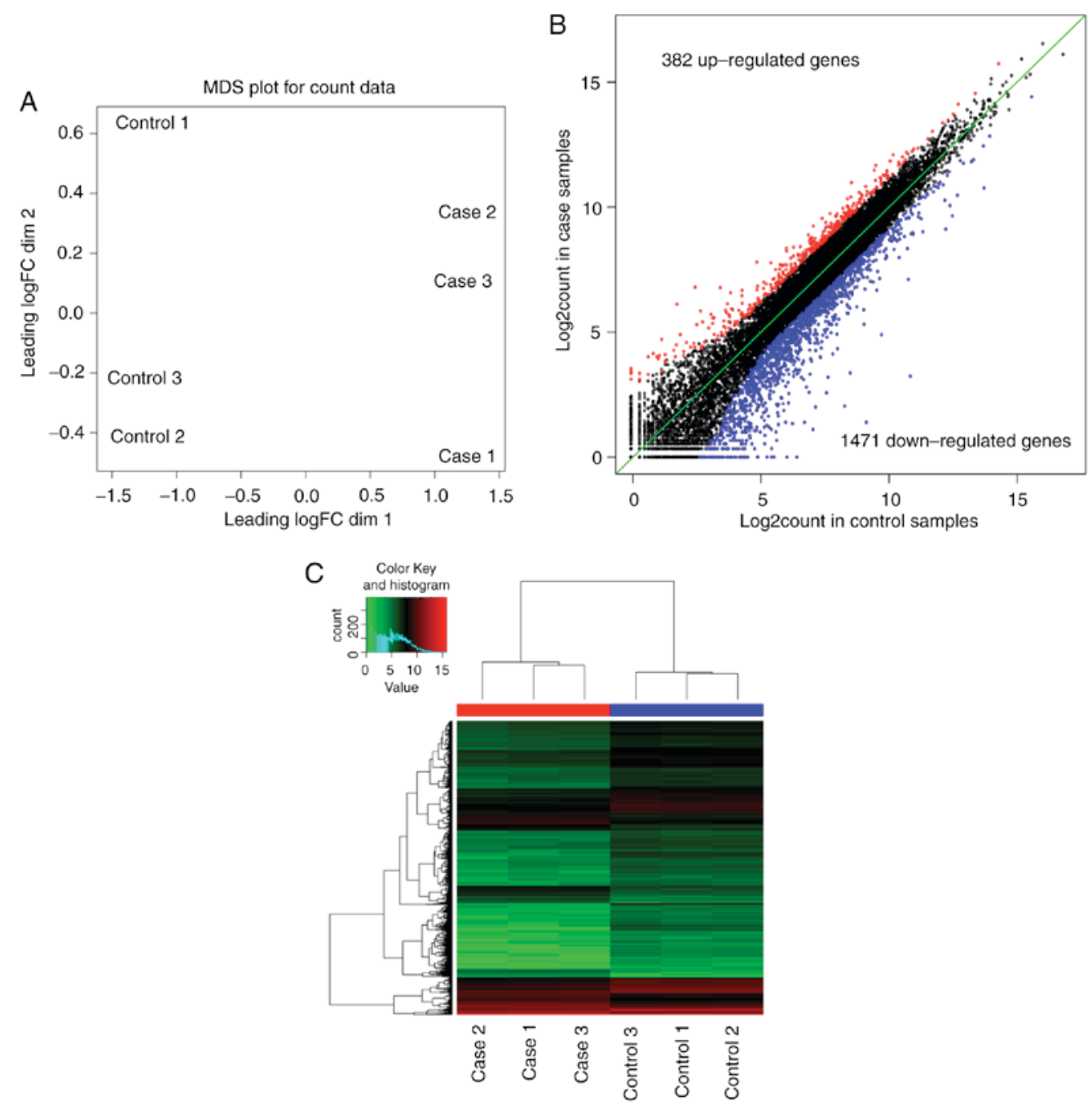

Figure 1. DEGs between 3 expression profiles from A549 cells with FOXD3 knockout and 3 from normal A549 cells. (A) Hierarchical clustering analysis of the 6 samples by MDS; (B) the fold change trend of the DEGs; and (C) the hierarchical clustering analysis of the samples based on the expression of the DEGs. DEGs, differentially expressed genes; FOXD3, forkhead box D3; MDS, multidimensional scaling; DEG, differentially expressed gene; FC, fold change.

A

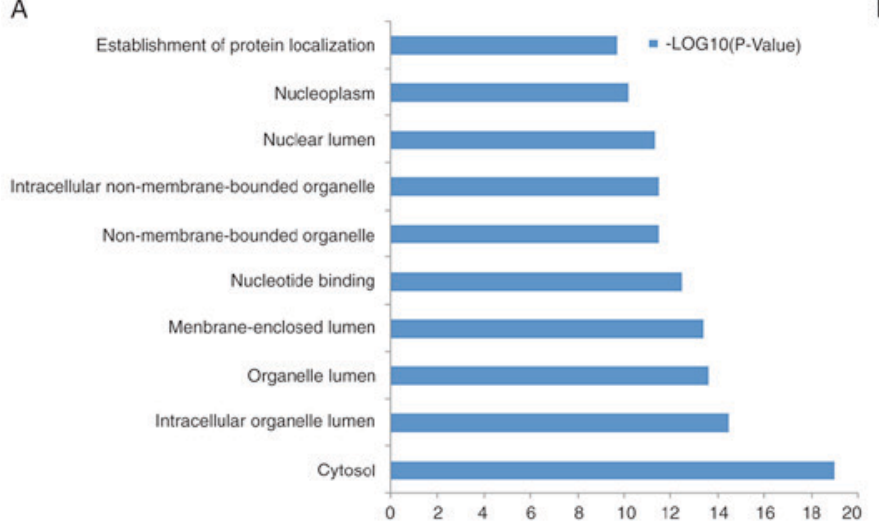

B

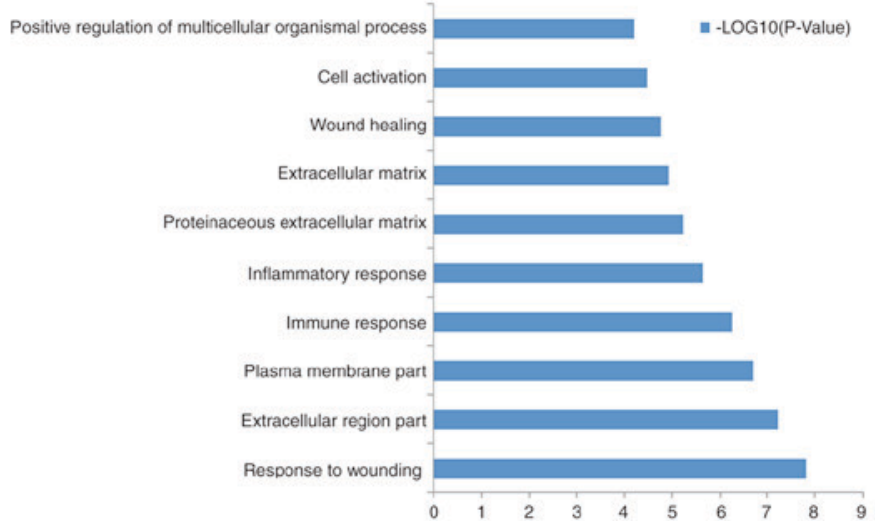

Figure 2. The top 10 GO terms in which (A) the DEGs, and (B) the alternative splicing genes were enriched. GO, Gene Ontology; DEGs, differentially expressed genes.

'NOD-like receptor signaling pathway'. The ASGs were predominately enriched in 'ubiquitin mediated proteolysis', 'chronic myeloid leukemia', 'aminoacyl-tRNA biosynthesis' and 'mTOR signaling pathway'.
PPI network. A total of 199 overlaps between the DEGs and the ASGs were identified, and the PPI network constructed from the 199 overlapping genes contained 97 nodes and 115 pairs (Fig. 3). Table IV lists the top 20 pairs with highest 
Table III. Enriched KEGG pathways for DEGs and ASGs between FOXD3-knockout and normal A549 cells.

A, Enriched KEGG pathways for DEGs

\begin{tabular}{lcc}
\hline Pathway name & Genes, $\mathrm{n}$ & P-value \\
\hline Graft-vs.-host disease & 12 & 0.0006 \\
Hematopoietic cell lineage & 18 & 0.0019 \\
NOD-like receptor signaling pathway & 14 & 0.0038 \\
ECM-receptor interaction & 17 & 0.0038 \\
Cell adhesion molecules & 23 & 0.0044 \\
Allograft rejection & 10 & 0.0045 \\
Cytokine-cytokine receptor interaction & 38 & 0.0055 \\
Glycine, serine and threonine metabolism & 9 & 0.0059 \\
MAPK signaling pathway & 38 & 0.0075 \\
p53 signaling pathway & 14 & 0.0085 \\
Natural killer cell mediated cytotoxicity & 22 & 0.0100 \\
Toll-like receptor signaling pathway & 18 & 0.0106 \\
Viral myocarditis & 14 & 0.0122 \\
Nitrogen metabolism & 7 & 0.0157 \\
Arginine and proline metabolism & 11 & 0.0217 \\
Complement and coagulation cascades & 13 & 0.0230 \\
Pathways in cancer & 42 & 0.0266 \\
Axon guidance & 20 & 0.0274 \\
Pathogenic Escherichia coli infection & 11 & 0.0344 \\
B cell receptor signaling pathway & 13 & 0.0413 \\
Small cell lung cancer & 14 & 0.0438 \\
\hline & & \\
\hline
\end{tabular}

B, Enriched KEGG pathways for ASGs

\begin{tabular}{lcc}
\hline Pathway name & Gene, $\mathrm{n}$ & P-value \\
\hline Ubiquitin mediated proteolysis & 33 & 0.0002 \\
Chronic myeloid leukemia & 20 & 0.0014 \\
Aminoacyl-tRNA biosynthesis & 13 & 0.0029 \\
mTOR signaling pathway & 15 & 0.0032 \\
Renal cell carcinoma & 18 & 0.0040 \\
Pancreatic cancer & 18 & 0.0054 \\
Neurotrophin signaling pathway & 26 & 0.0079 \\
Pathways in cancer & 55 & 0.0133 \\
Ribosome & 19 & 0.0173 \\
Pyrimidine metabolism & 20 & 0.0208 \\
Acute myeloid leukemia & 14 & 0.0215 \\
Small cell lung cancer & 18 & 0.0250 \\
Wnt signaling pathway & 28 & 0.0276 \\
Cell cycle & 24 & 0.0297 \\
VEGF signaling pathway & 16 & 0.0373 \\
Lysine degradation & 11 & 0.0377 \\
Insulin signaling pathway & 25 & 0.0387 \\
Glioma & 14 & 0.0403 \\
Prostate cancer & 18 & 0.0414 \\
Lysosome & 22 & 0.0465
\end{tabular}

Table III. Continued.

\begin{tabular}{lcc}
\hline Pathway name & Genes, $\mathrm{n}$ & P-value \\
\hline Endometrial cancer & 12 & 0.0484 \\
N-glycan biosynthesis & 11 & 0.0495
\end{tabular}

KEGG, Kyoto Encyclopedia of Genes and Genomes; DEG, differentially expressed genes; ASG, alternative splicing gene; NOD, nucleotide oligomerization; ECM, extracellular matrix; MAPK, mitogen-activated protein kinase; mTOR, mechanistic target of rapamycin; tRNA, transfer RNA; VEGF, vascular endothelial proliferation factor

combined scores, and Table $\mathrm{V}$ lists the top 10 nodes according to connectivity degree.

\section{Discussion}

Lung cancer is a serious threat to human health and survival(22). Despite progress in diagnosis and treatment, the 5-year survival rate of patients with lung cancer is only 9-20\% (23). FOXD3 has been suggested to be a tumor suppressor in various types of cancer (8-10). However, the underlying mechanism of FOXD3 activity in lung cancer remains unclear. In the present study, DEGs and ASGs between FOXD3-knockout and normal lung cancer A549 cells were identified, and functional enrichment analysis was performed to identify the associated biological processes involved in lung cancer. Finally, a PPI network of the most significant genes was constructed. These results may contribute to the understanding of the role of FOXD3 in lung cancer.

The most enriched GO terms for the DEGs were 'response to wounding', 'extracellular region', 'plasma membrane' and 'immune response'. The ASGs were mainly enriched in 'cytosol', 'intracellular organelle lumen', 'organelle lumen' and 'membrane-enclosed lumen' (Fig. 2). The wound response involves clotting and coagulation, tissue remodeling, cellular migration and proliferation, and angiogenesis (24). The majority of these processes also serve important roles in the progression of cancer. One study reported that the upregulation of factors associated with the 'wound response' term was highly prognostic of breast cancer survival, and revealed a strong association between the pathogenic conditions identified by this signature and those identified using serum-treated fibroblasts (25). In lung cancer, the upregulation of genes associated with the 'wound response' term has been demonstrated as predictive of poor overall survival time and increased risk of metastasis (26).

The cell membrane is a biological membrane that separates the interior of cells from the outside environment (27). Plasma membrane fluidity depends on the composition of the lipids and proteins in the membrane, and has been demonstrated to be significantly associated with the malignant potential of cancer cells (28), with alterations in the plasma membrane fluidity of cancer cells associated with their capacity to form metastases (29). In lung cancer, studies reported that patients with high plasma membrane fluidity had poorer prognoses than those with less fluid membranes, 


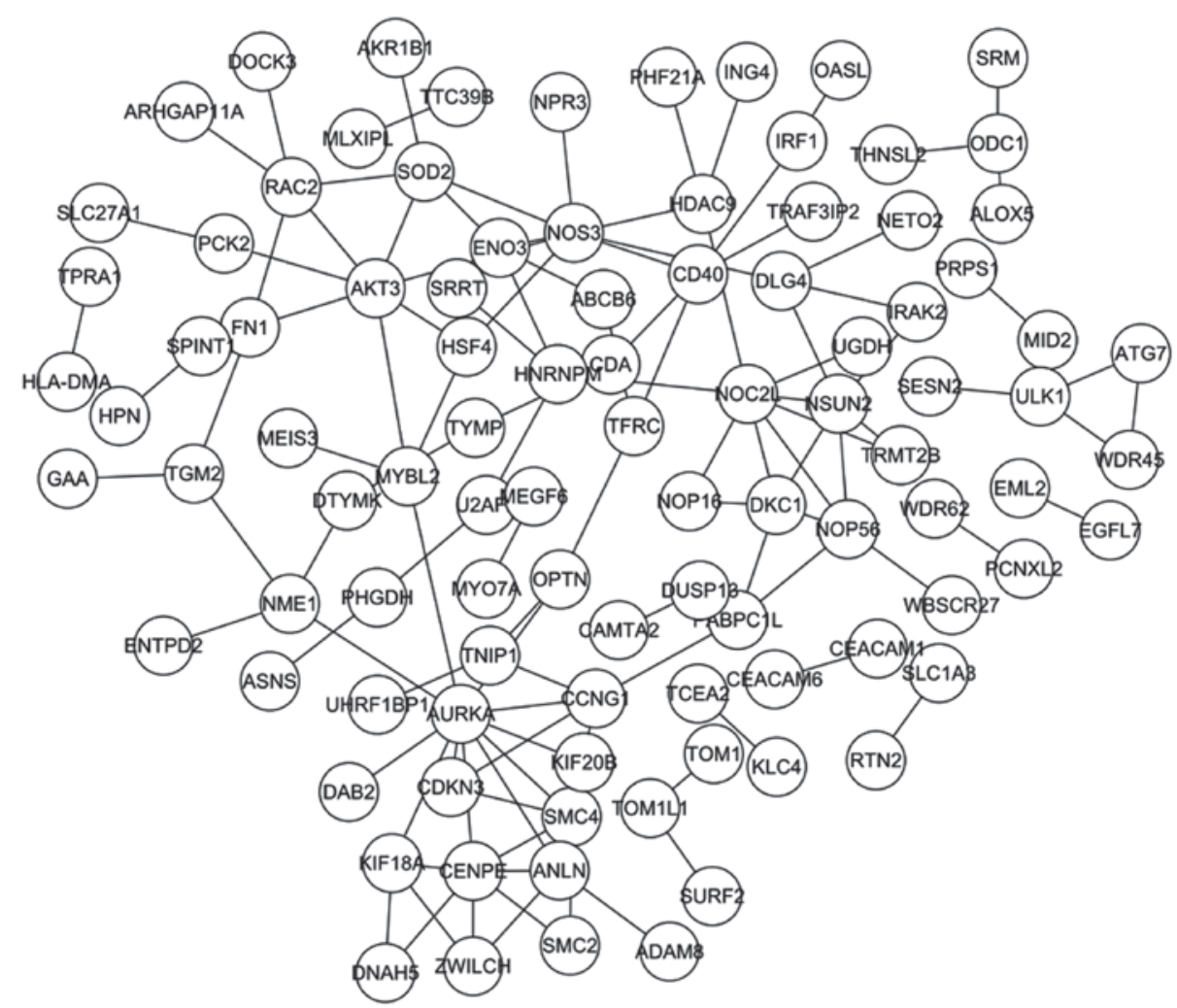

Figure 3. PPI network demonstrating the interactions between overlapping DEGs and alternative splicing genes. PPI, protein-protein interaction; DEGs, differentially expressed genes.

Table IV. Top 20 pairs of the protein-protein interaction network as determined by the highest combined score.

\begin{tabular}{llc}
\hline Gene 1 & Gene 2 & Combined score \\
\hline SMC4 & SMC2 & 0.999 \\
NOP56 & DKC1 & 0.997 \\
TFRC & OPTN & 0.994 \\
SRM & ODC1 & 0.989 \\
CDA & TYMP & 0.987 \\
HNRNPM & U2AF2 & 0.979 \\
ZWILCH & CENPE & 0.977 \\
CEACAM6 & CEACAM1 & 0.970 \\
TGM2 & FN1 & 0.970 \\
NOS3 & AKT3 & 0.964 \\
DTYMK & NME1 & 0.954 \\
CENPE & KIF18A & 0.946 \\
NOP56 & NOC2L & 0.941 \\
ATG7 & ULK1 & 0.937 \\
ARHGAP11A & RAC2 & 0.936 \\
ZWILCH & KIF18A & 0.930 \\
OASL & IRF1 & 0.925 \\
HDAC9 & PHF21A & 0.925 \\
HDAC9 & NOS3 & 0.923 \\
DKC1 & NOC2L & 0.911 \\
\hline
\end{tabular}

and the fluidity variable may be used as an independent additional prognostic factor $(28,30,31)$.
Table V. The top 10 nodes of the protein-protein interaction network as determined by the highest connectivity degree.

\begin{tabular}{lc}
\hline Gene & Degree \\
\hline AURKA & 11 \\
NOS3 & 8 \\
NOC2L & 8 \\
CENPE & 7 \\
AKT3 & 7 \\
NSUN2 & 6 \\
SOD2 & 5 \\
SMC4 & 5 \\
RAC2 & 5 \\
NOP56 & 5 \\
\hline
\end{tabular}

Cytosol is the fluid within cells, a component essential to the process of cytokinesis, a critical stage in cell proliferation $(32,33)$. Another major function of cytosol is to transport metabolites; most tumor cells demonstrate different metabolic pathways to normal cells (34). One study indicated that metabolism contributed to the tumor proliferation, migration, and metastasis of lung cancer (35).

Other enriched GO terms, e.g., 'organelle lumen', have also been associated with tumorigenesis. Jingye et al (36) reported that a disordered $\mathrm{pH}$ in the organelle lumen is a common characteristic of cancer cells. Despite a number of studies reporting the FOXD3-mediated inhibition of the growth, invasion and 
migration of tumor cells in various types of cancer, including lung cancer (37-39), limited data is available regarding the association between FOXD3 and these GO terms. As discussed, the identified GO terms have been associated with the growth, invasion and migration of tumor cells, thus it is speculated that FOXD3 may affect the progression of lung cancer indirectly by regulating these biological processes.

From the identified KEGG pathways, the mechanistic target of rapamycin (mTOR) signaling pathway has also been associated with the growth and proliferation of tumor cells, and the deregulation of multiple elements of the mTOR pathway has been reported in numerous types of cancer (40). The NOD-like receptor signaling pathway is involved in the formation of inflammasomes, and numerous types of cancer are associated with inflamed tissue (41). However, the associations between FOXD3 and the identified KEGG pathways require further exploration.

A total of 199 overlaps between the DEGs and the ASGs were identified, from which the PPI network was constructed (Fig. 3). The top 5 nodes of the PPI network, with the highest degree, were aurora kinase A (AURKA), nitric oxide synthase 3 (NOS3), NOC2-like nucleolar associated transcriptional repressor $(N O C 2 L)$, centromere protein $\mathrm{E}(C E N P E)$ and $A K T 3$. The majority of these genes have been previously associated with tumorigenesis. AURKA and NOS3 serve important roles in the development of various types of cancer, including lung cancer; AURKA is a cell cycle-regulated kinase involved in spindle formation and chromosome segregation (42). Various types of cancer exhibit the overexpression of AURKA, which is associated with chromosomal instability, centrosomal amplification/aneuploidy, therapeutic resistance, cell-cycle progression and anti-apoptosis. As an oncogene, AURKA is an important therapeutic target in lung cancer, and cell proliferation, apoptosis and cell cycle progression are associated with the expression of AURKA (43). NOS3 encodes an enzyme that regulates the production of nitric oxide and contributes to uncontrollable cell growth in a number of cancer types (44). Various studies have demonstrated associations between NOS3 and cancer processes. For example, Arıkan et al (45) reported that the NOS3 Glu298Asp polymorphism may be associated with the risk and progression of colorectal cancer. Lee et al (46) reported that genetic polymorphisms in NOS3 modified individual susceptibility to invasive breast cancer with lymph node involvement in Korean women. Furthermore, the expression of NOS3 has been reported to contribute to the tumor angiogenesis and lymph metastasis of human non-small cell lung cancer (47).

The expression of other genes, including CENPE, NOC2L and $A K T 3$ has also been associated with tumorigenesis (48-50). $C E N P E$ was identified as a novel therapeutic candidate in neuroblastoma (50), and the selective activation of the AKT3 protein promoted cell survival and tumor development in non-familial melanomas in one study (48). To the best of our knowledge, there is no experimental evidence of the direct association between FOXD3 and these genes. However, the biological functions associated with these genes in the context of cancer correspond with the regulating mechanism of FOXD3 in lung cancer. FOXD3 acts as a tumor suppressor by regulating the expression of the target genes, thus inhibiting the growth, invasion and migration of tumor cells (51). Few specific targets for FOXD3 in lung cancer have been reported, whereas AURKA and NOS3 serve critical roles in the growth, invasion and migration of tumor cells in lung cancer. Therefore, we speculate that $A U R K A$ and NOS3 may be the targets of $F O X D 3$ that execute its effect in lung cancer. Confirmation of these conclusions and further exploration of the specific mechanism of FOXD3 regulation in lung cancer are required.

In conclusion, FOXD3 serves an important role in regulating the growth, migration and proliferation of lung cancer cells. Genes such as AURKA and NOS3 may be targets of $F O X D 3$, mediating its effect in lung cancer. The present study contributes to the existing understanding of the molecular mechanism of lung cancer and may provide data to contribute towards novel strategies for improving the diagnosis and therapy of lung cancer.

\section{Acknowledgements}

The present study was supported by the Municipal Science and Technology Commission of Tianjin (grant nos. 15ZLZLZF00440 and 16ZLZXZF00120) and the Health Bureau Science and Technology Foundation of Tianjin (grant no. 2014KZ102).

\section{References}

1. Reck M,Heigener DF,Mok T,Soria JC and Rabe KF: Management of non-small-cell lung cancer: Recent developments. Lancet 382: 709-719, 2013.

2. Grunnet $\mathrm{M}$ and Sorensen JB: Carcinoembryonic antigen (CEA) as tumor marker in lung cancer. Lung Cancer 76: 138-143, 2012.

3. Lozano R, Naghavi M, Foreman K, Lim S, Shibuya K, Aboyans V, Abraham J, Adair T, Aggarwal R, Ahn SY, et al: Global and regional mortality from 235 causes of death for 20 age groups in 1990 and 2010: A systematic analysis for the Global Burden of disease study 2010. Lancet 380: 2095-2128, 2012.

4. Wang Y, Chen J, Wu S, Hu C, Li X, Wang Y, Yang Y, Rajan N, Chen Y, Chen Y, et al: Clinical effectiveness and clinical toxicity associated with platinum-based doublets in the first-line setting for advanced non-squamous non-small cell lung cancer in Chinese patients: A retrospective cohort study. BMC Cancer 14: 940, 2014.

5. Cooper WA, Lam DC, O'Toole SA and Minna JD: Molecular biology of lung cancer. Lung Cancer 42: 378-386, 2004.

6. Weigel D and Jäckle H: The fork head domain: A novel DNA binding motif of eukaryotic transcription factors? Cell 63: 455-456, 1990.

7. Sutton J, Costa R, Klug M, Field L, Xu D, Largaespada DA, Fletcher CF, Jenkins NA, Copeland NG, Klemsz M and Hromas R: Genesis, a winged helix transcriptional repressor with expression restricted to embryonic stem cells. J Biol Chem 271: 23126-23133, 1996.

8. Yan JH, Zhao CL, Ding LB and Zhou X: FOXD3 suppresses tumor growth and angiogenesis in non-small cell lung cancer. Biochem Biophys Res Commun 466: 111-116, 2015.

9. Cheng AS, Li MS, Kang W, Cheng VY, Chou JL, Lau SS Go MY, Lee CC, Ling TK, Ng EK, et al: Helicobacter pylori causes epigenetic dysregulation of FOXD3 to promote gastric carcinogenesis. Gastroenterology 144: 122-133.e9, 2013.

10. Abel EV and Aplin AE: FOXD3 is a mutant B-RAF-regulated inhibitor of $\mathrm{G}(1)-\mathrm{S}$ progression in melanoma cells. Cancer Res 70: 2891-2900, 2010.

11. Katiyar P and Aplin AE: FOXD3 regulates migration properties and Rnd3 expression in melanoma cells. Mol Cancer Res 9: 545-552, 2011.

12. Li D, Mei H, Qi M, Yang D, Zhao X, Xiang X, Pu J, Huang K, Zheng L and Tong Q: FOXD3 is a novel tumor suppressor that affects growth, invasion, metastasis and angiogenesis of neuroblastoma. Oncotarget 4: 2021-2044, 2013.

13. Kim D, Pertea G, Trapnell C, Pimentel H, Kelley R and Salzberg SL: TopHat2: Accurate alignment of transcriptomes in the presence of insertions, deletions and gene fusions. Genome Biol 14: R36, 2013. 
14. Anders S, Pyl PT and Huber W: HTSeq-a Python framework to work with high-throughput sequencing data. Bioinformatics 31 : $166-169,2015$.

15. Culpepper SA and Aguinis HR: Is for Revolution: A Cutting-Edge, free, open source statistical package. Organizational Res Methods 13: 735-740, 2011.

16. Warnes GR, Bolker B, Bonebakker L, et al: Gplots: Various $\mathrm{R}$ programming tools for plotting data. R package version 2.12. 1. Available online at: http://CRAN. R-project. org/package $=$ gplots.

17. Shen S, Park JW, Lu ZX, Lin L, Henry MD, Wu YN, Zhou Q and Xing Y: rMATS: Robust and flexible detection of differential alternative splicing from replicate RNA-Seq data. Proc Natl Acad Sci USA 111: E5593-E5601, 2014.

18. Dennis G Jr, Sherman BT, Hosack DA, Yang J, Gao W, Lane HC and Lempicki RA: DAVID: Database for Annotation, Visualization, and Integrated Discovery. Genome Biol 4: P3, 2003.

19. Szklarczyk D, Franceschini A, Kuhn M, Simonovic M, Roth A, Minguez P, Doerks T, Stark M, Muller J, Bork P, et al: The STRING database in 2011: Functional interaction networks of proteins, globally integrated and scored. Nucleic Acids Res 39 (Database issue): D561-D568, 2011.

20. Franceschini A, Szklarczyk D, Frankild S, Kuhn M, Simonovic M, Roth A, Lin J, Minguez P, Bork P, von Mering C and Jensen LJ: STRING v9.1: Protein-protein interaction networks, with increased coverage and integration. Nucleic Acids Res 41 (Database issue): D808-D815, 2013.

21. Smoot ME, Ono K, Ruscheinski J, Wang PL and Ideker T: Cytoscape 2.8: New features for data integration and network visualization. Bioinformatics 27: 431-432, 2011.

22. Jemal A, Bray F, Center MM, Ferlay J, Ward E and Forman D: Global cancer statistics. CA Cancer J Clin 61: 69-90, 2011.

23. Yang P: Epidemiology of lung cancer prognosis: Quantity and quality of life. Methods Mol Biol 471: 469-86, 2009.

24. Schäfer M and Werner S: Cancer as an overhealing wound: An old hypothesis revisited. Nat Rev Mol Cell Biol 9: 628-638, 2008.

25. Troester MA, Lee MM, Carter M, Fan C, Cowan DW, Perez ER, Pirone JR, Perou CM, Jerry DJ and Schneider SS: Activation of host wound responses in breast cancer microenvironment. Clin Cancer Res 15: 7020-7028, 2009.

26. Chang HY, Nuyten DS, Sneddon JB, Hastie T, Tibshirani R, Sørlie T, Dai H, He YD, van't Veer LJ, Bartelink H, et al: Robustness, scalability, and integration of a wound-response gene expression signature in predicting breast cancer survival. Proc Natl Acad Sci USA 102: 3738-3743, 2005.

27. Singleton P: Bacteria in Biology, Biotechnology, and Medicine. 5th edition, John Wiley, Hoboken, NJ, pp444-454, 1999.

28. Sok M, Sentjurc M, Schara M, Stare J and Rott T: Cell membrane fluidity and prognosis of lung cancer. Ann Thorac Surg 73: $1567-1571,2002$

29. Nakazawa I and Iwaizumi M: A role of the cancer cell membrane fluidity in the cancer metastases: An ESR study. Tohoku J Exp Med 157: 193-198, 1989.

30. Deliconstantinos G: Physiological aspects of membrane lipid fluidity in malignancy. Anticancer Res 7: 1011-1021, 1987.

31. Nakazawa I and Iwaizumi M: A role of the cancer cell membrane fluidity in the cancer metastases: Aan ESR study. Tohoku J Exp Med 157: 193-198, 1989.

32. Winey M, Mamay CL, O'Toole ET, Mastronarde DN, Giddings TH Jr, McDonald KL and McIntosh JR: Three-dimensional ultrastructural analysis of the Saccharomyces cerevisiae mitotic spindle. J Cell Biol 129: 1601-1615, 1995.

33. Jana SS, Kawamoto S and Adelstein RS: A specific isoform of nonmuscle myosin II-C is required for cytokinesis in a tumor cell line. J Biol Chem 281: 24662-24670, 2006.

34. Weisiger RA: Cytosolic fatty acid binding proteins catalyze two distinct steps in intracellular transport of their ligands. Mol Cell Biochem 239: 35-43, 2002.
35. Li XB, Gu JD and Zhou QH: Review of aerobic glycolysis and its key enzymes-new targets for lung cancer therapy. Thoracic Cancer 6: 17-24, 2015.

36. Jingye Z, Zining L, Peng L, Jun Q, Xinwei L, Lu W, Wei F, Liang C, Xunbin W and Cong L: Selective imaging and cancer cell death via $\mathrm{pH}$ switchable near-infrared fluorescence and photothermal effects. Chem Sci 7: 5995-6005, 2016.

37. Ackermann S, Kocak H, Hero B, Ehemann V, Kahlert Y, Oberthuer A, Roels F, Theißen J, Odenthal M, Berthold F and Fischer M: FOXP1 inhibits cell growth and attenuates tumorigenicity of neuroblastoma. Bmc Cancer 14: 840, 2014.

38. Kos R, Reedy MV, Johnson RL and Erickson CA: The winged-helix transcription factor FoxD3 is important for establishing the neural crest lineage and repressing melanogenesis in avian embryos. Development 128: 1467-1479, 2001.

39. Wang C, Huang Y and Dai W: Tumor suppression function of FoxD3 in lung cancer. Ir J Med Sci 185: 547-553, 2016.

40. Pópulo H, Lopes JM and Soares P: The mTOR signalling pathway in human cancer. Int J Mol Sci 13: 1886-1918, 2012.

41. Castaño-Rodríguez N, Kaakoush NO, Goh KL, Fock KM and Mitchell HM: The NOD-like receptor signalling pathway in Helicobacter pylori infection and related gastric cancer: A case-control study and gene expression analyses. PLoS One 9: e98899, 2014

42. Lens SM, Voest EE and Medema RH: Shared and separate functions of polo-like kinases and aurora kinases in cancer. Nat Rev Cancer 10: 825-841, 2010.

43. Ma ZL, Zhang BJ, Wang DT, Li X, Wei JL Zhao BT, Jin Y, $\mathrm{Li}$ YL and Jin YX: Tanshinones suppress AURKA through up-regulation of miR-32 expression in non-small cell lung cancer. Oncotarget 6: 20111-20120, 2015.

44. Xu W, Charles IG, Moncada S, Gorman P, Sheer D, Liu L and Emson P: Mapping of the genes encoding human inducible and endothelial nitric oxide synthase (NOS2 and NOS3) to the pericentric region of chromosome 17 and to chromosome 7 , respectively. Genomics 21: 419-422, 1994.

45. Arıkan S, Cacina C, Guler E, Culcu S, Tuna G and Yaylımeraltan I: The effects of NOS3 Glu298Asp variant on colorectal cancer risk and progression in Turkish population. Mol Biol Rep 39: 3245-3249, 2012

46. Lee KM, Choi JY, Lee JE, Noh DY, Ahn SH, Han W, Yoo KY, Hayes RB and Kang D: Genetic polymorphisms of NOS3 are associated with the risk of invasive breast cancer with lymph node involvement. Breast Cancer Res Treat 106: 433-438, 2007.

47. Wang JH, Chen LB, Heng-Hui MA and Meng K: Expressions of NOS2 and NOS3 in human non-small cell lung cancer and the relationship with tumor angiogenesis and lymph node metastasis. J Med Postgraduates, 2004.

48. Stahl JM, Sharma A, Cheung M, Zimmerman M, Cheng JQ, Bosenberg MW, Kester M, Sandirasegarane L and Robertson GP: Deregulated Akt3 activity promotes development of malignant melanoma. Cancer Res 64: 7002-7010, 2004.

49. Terasaka Y, Miyazaki D, Yakura K, Haruki T and Inoue Y: Induction of IL-6 in transcriptional networks in corneal epithelial cells after herpes simplex virus type 1 infection. Invest Ophthalmol Vis Sci 51: 2441-2449, 2010.

50. Balamuth NJ, Wood A, Wang Q, Jagannathan J, Mayes P, Zhang Z, Chen Z, Rappaport E, Courtright J, Pawel B, et al: Serial transcriptome analysis and cross-species integration identifies centromere-associated protein $\mathrm{E}$ as a novel neuroblastoma target. Cancer Res 70: 2749-2758, 2010.

51. Weiss MB, Abel EV, Dadpey N and Aplin AE: FOXD3 modulates migration through direct transcriptional repression of TWIST1 in melanoma. Mol Cancer Res 12: 1314-1323, 2014. 